\title{
CT Use in Acute Pulmonary Thromboembolism
}

\section{Dr Sunil Kumar Naramnatha}

College \& General Hospital, Rajahmundry, India

\section{Introduction}

- CT pulmonary angiography has become the "gold standard" for the diagnosis of acute pulmonary thromboembolism and has replaced catheter pulmonary angiography and ventilation- perfusion scintigraphy as the first-line imaging method.

- Current pulmonary CTA techniques involve lower doses of contrast medium and radiation along with advanced post processing applications to enhance image quality, diagnostic accuracy, and provide added value in patient management.

\section{Objectives}

To evaluate the role of CT in diagnosis of acute pulmonary thromboembolism \& also to differentiate it from chronic pulmonary thromboembolism.

\section{Materials \& Methods}

A retrospective study was performed on patients with clinical suspicion of acute pulmonary thromboembolism who underwent chest CT at the Department of Radio diagnosis. All patients were scanned on Toshiba Alexion 16 slice. Plain \&contrast studies were performed.

\section{Observation \& Results}

- CT Findings of Acute Pulmonary Thromboembolism:

- CT criteria for acute pulmonary embolism are complete or partial filling defect in pulmonary arteries or its branches.

- Railway track sign: Endoluminal clot surrounded by rim of contrast material.

- Polo mint sign : central filling defect represent the thrombus while the peripheral rim appears as a hyperattenuating area due to contrast which mimics the polo mint.

- Indirect Signs: Peripheral areas of consolidation or ground glass opacities resulting from pulmonary hemorrhage or infarction \& pleural effusion.

- Reverse halo sign: An area of ground glass opacity surrounded by rim of consolidation seen in pulmonary infarction.

\subsection{Acute Pulmonary Thromboembolism}

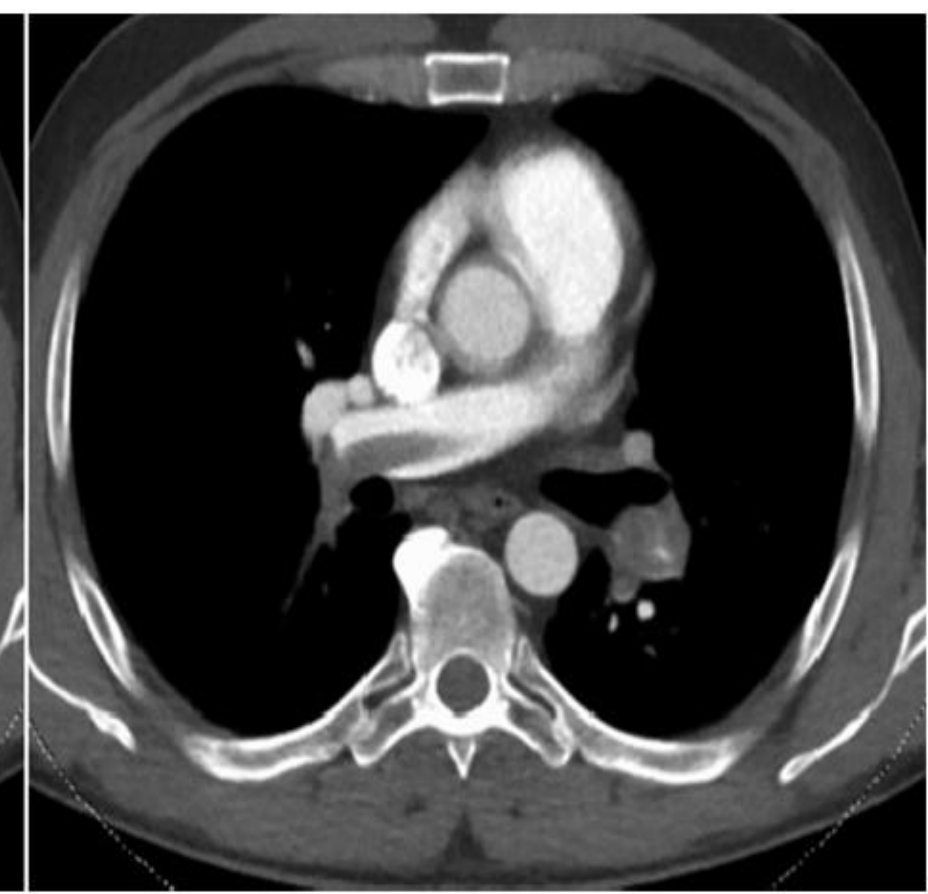

Axial CECT shows central saddle embolus at bifurca tion of main pulmonary arteries 


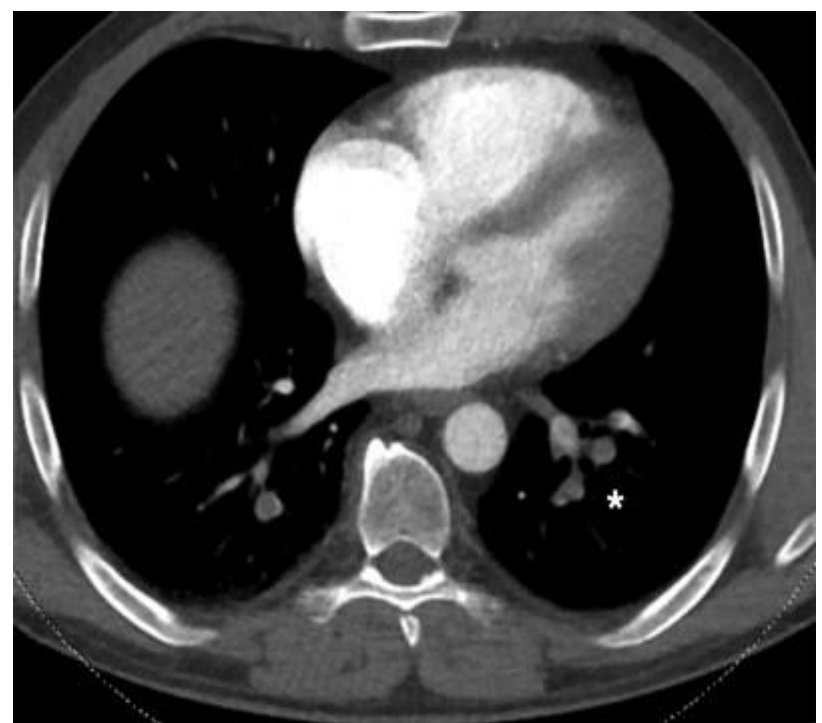

Axial CECT show bilateral segmental and subsegmental thrombi

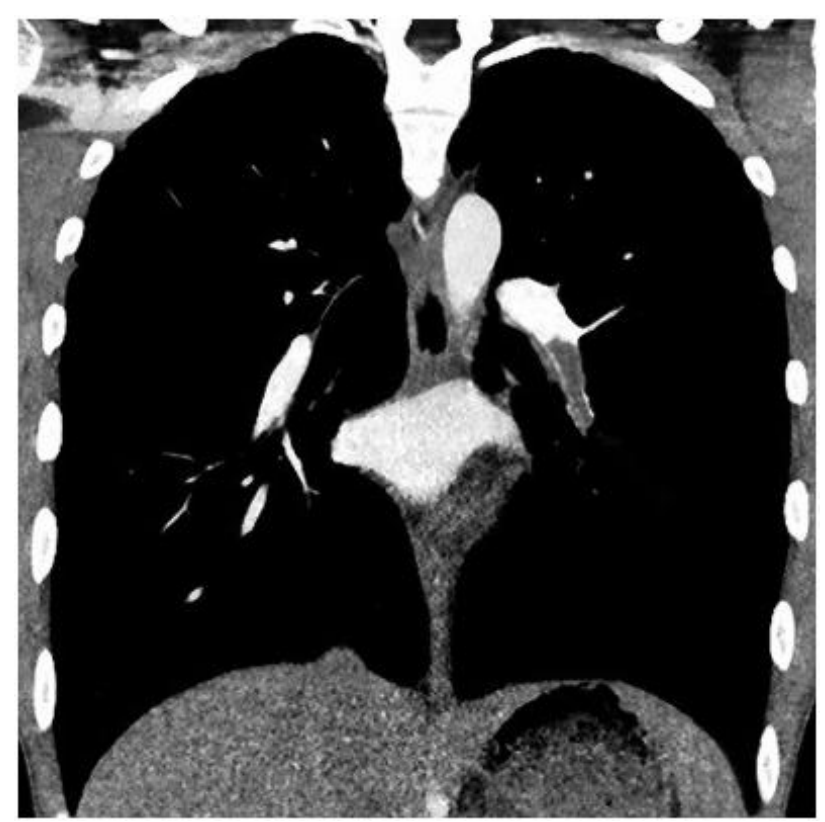

Coronal reconstruction images show filling defect in left lower lobe pulmonary artery

\section{CT Features of Chronic Pulmonary Thromboembolism}

Pulmonary Arterial Signs:

- Complete Obstruction: complete vessel cutoff results in a convex margin of the contrast material bolus- "pouch defect".

- Partial Obstruction: webs, bands, focal stenotic vessel segment \& post stenotic dilatation.

- Collateral vessels: Bronchial artery dilatation( $>2 \mathrm{~mm}) \&$ tortuosity.

- Signs of pulmonary artery hypertension Parenchymal Signs:

- Scars from prior pulmonary infarctions: areas of decreased lung attenuation.

- Mosaic perfusion: sharply demarcated regions of decreased and increased attenuation.

- Cylindrical bronchial airway dilatation

\subsection{Chronic Pulmonary Thromboembolism}

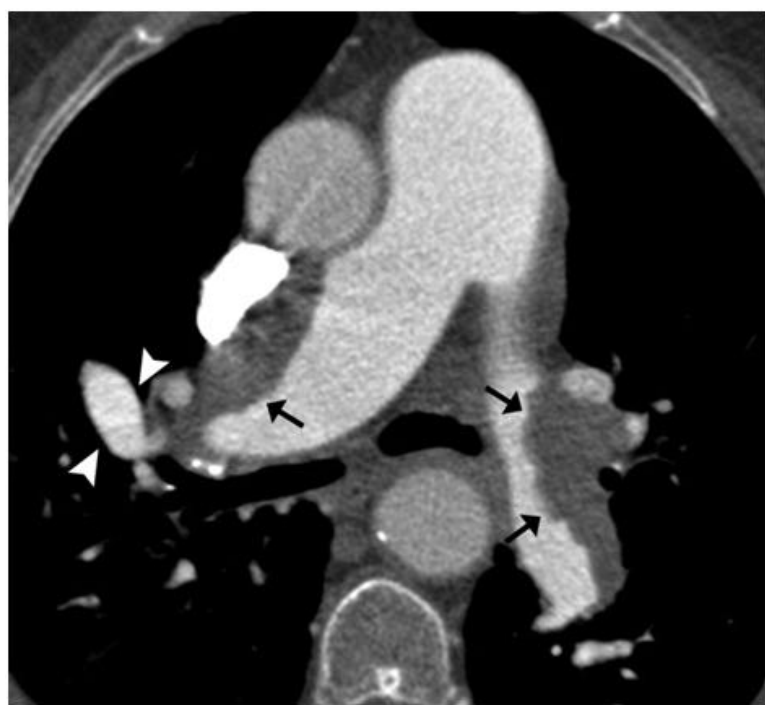

Axial CECT scan shows bilateral eccentric chronic thrombi producing irregular contours of the intimal surface of both main pulmonary arteries and poststenotic dilatation in the posterior segmental artery of the right upper lobe

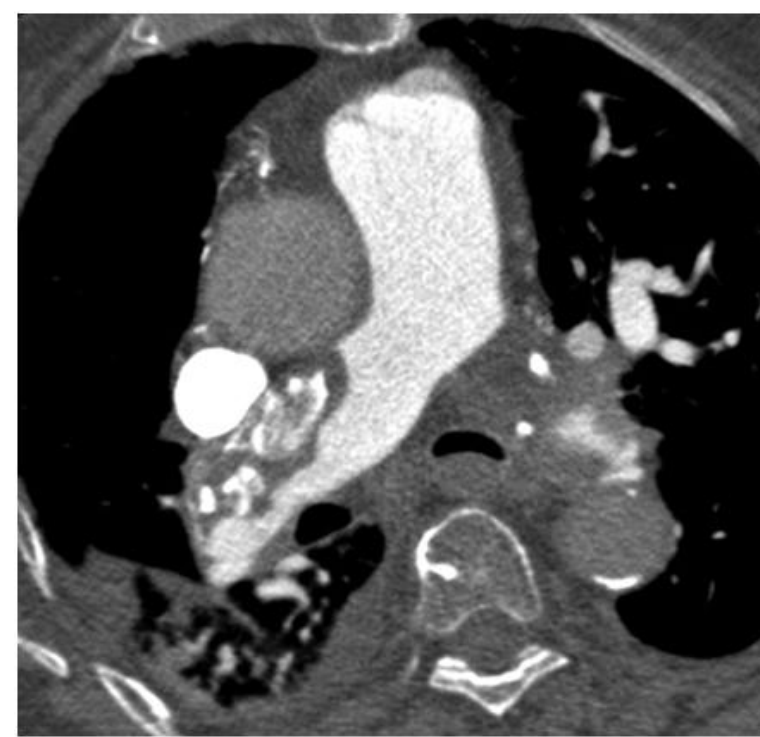

Axial CECT scan shows a partially calcified thrombus in the right pulmonary artery

Differentiation of Acute \& Chronic Pulmonary

\begin{tabular}{|c|c|c|}
\hline \multicolumn{3}{|c|}{ Thromboembolism } \\
\hline CT Findings & Acute & Chronic \\
\hline $\begin{array}{l}\text { Vascular } \\
\text { Signs }\end{array}$ & $\begin{array}{l}\text { Central or eccentric } \\
\text { filling defect; acute } \\
\text { angle with vessel } \\
\text { wall; preserved } \\
\text { caliber of artery. }\end{array}$ & $\begin{array}{c}\text { Peripheral crescent shaped } \\
\text { filling defect; obtuse angle } \\
\text { with vessel wall; vessel } \\
\text { narrowing. }\end{array}$ \\
\hline $\begin{array}{l}\text { Collateral } \\
\text { vessels }\end{array}$ & Absent & $\begin{array}{c}\text { Bronchial \& non- } \\
\text { bronchial systemic arteries } \\
\text { dilatation. }\end{array}$ \\
\hline $\begin{array}{c}\text { Signs of } \\
\text { Pulmonary } \\
\text { hypertension }\end{array}$ & May be present & Commonly present \\
\hline $\begin{array}{c}\text { Parenchymal } \\
\text { signs }\end{array}$ & $\begin{array}{l}\text { Pulmonary infarction } \\
\text { (reverse halo sign). }\end{array}$ & $\begin{array}{l}\text { Pulmonary scars from } \\
\text { prior infarction. Mosaic } \\
\text { attenuation. }\end{array}$ \\
\hline
\end{tabular}




\section{Conclusion}

a) The timely and accurate diagnosis of acute pulmonary thromboembolism is crucial to providing appropriate patient care.

b) Pulmonary CT angiography is the imaging modality of choice in suspected acute pulmonary thromboembolism.

\section{References}

[1] Moritz H. Albrecht, Matthew W. Bickford, John W. Nance, Jr., Longjiang Zhang, Carlo N. De Cecco, Julian L. Wichmann, Thomas J. Vogl, and U. Joseph Schoepf

[2] State-of-the-Art Pulmonary CT Angiography for Acute Pulmonary Embolism, American Journal of Roentgenology 2017 208:3, 495-504

[3] Eva Castañer, Xavier Gallardo, Eva Ballesteros, Marta Andreu, Yolanda Pallardó, Josep Maria Mata, and Lluis Riera, CT Diagnosis of Chronic Pulmonary Thromboembolism, RadioGraphics 2009 29:1, 31-50

[4] Conrad Wittram, Michael M. Maher, Albert J. Yoo, Mannudeep K. Kalra, Jo-Anne O. Shepard, and Theresa C. McLoud, CT Angiography of Pulmonary Embolism: Diagnostic Criteria and Causes of Misdiagnosis, RadioGraphics 2004 24:5, 1219-1238 